\title{
Supporting Information: Photoinduced Homolytic Bond Cleavage of the Central Si-C Bond in Porphyrinic Macrocycles is a Charge Polarization Driven Process
}

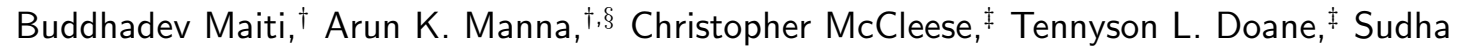 \\ Chakrapani, Clemens Burda, ${ }^{*, \ddagger}$ and Barry D. Dunietz ${ }^{*, \dagger}$ \\ Department of Chemistry and Biochemistry, Kent State University, Kent, OH 44242, USA, Department of \\ Chemistry, Case Western Reserve University, Cleveland, OH 44106, USA, and Department of Physiology \\ and Biophysics, Case Western Reserve University, Cleveland, OH 44106, USA \\ Received September 15, 2016; E-mail: burda@cwru.edu,216-368-5918,f:216-368-3006; \\ bdunietz@kent.edu,330-672-2032,f:330-672-3816
}

${ }^{\dagger}$ Kent State University, OH

${ }^{\ddagger}$ Case Western Reserve University, OH

${ }^{\top}$ Case Western Reserve University, OH

${ }^{\S}$ Work done at KSU, currently at Weizmann Inst. of Science, IL. 


\section{Supporting Information (SI)}

SI Table 1. Excitations energies of neutral, radical and ionic forms of Si-Pc Complex (eV) using $\omega$ B97XD functional with 6-311G(d) and $6-311+G(d, p)$ basis sets compared to B3LYP and with PCM representing methanol solvent. The main molecular orbital (MO) replacements are listed with their corresponding coefficients in parenthesis. ( $\mathrm{H}, \mathrm{S}$ and $\mathrm{L}$ denote HOMO, SOMO and LUMO.)

\begin{tabular}{|c|c|c|c|c|c|c|}
\hline \multicolumn{7}{|c|}{$\omega \mathrm{B} 97 \mathrm{XD} / 6-311 \mathrm{G}(\mathrm{d})$} \\
\hline Form & $\mathrm{E}_{Q}(\mathrm{eV})$ & MOs Configuration & $\mathrm{E}_{Q(S i)}(\mathrm{eV})$ & MOs Configuration & $\mathrm{E}_{B}(\mathrm{eV})$ & MOs Configuration \\
\hline \multirow[t]{2}{*}{$\mathrm{Si}-\mathrm{Pc}$} & $2.18(0.56)$ & $\mathrm{H} \rightarrow \mathrm{L}(0.90)$ & $2.82(0.00)$ & $\mathrm{H}-3 \rightarrow \mathrm{L}(0.89)$ & $3.41(0.24)$ & $\mathrm{H}-3 \rightarrow \mathrm{L}(0.73)$ \\
\hline & $2.18(0.56)$ & $\mathrm{H} \rightarrow \mathrm{L}+1(0.90)$ & $2.83(0.00)$ & $\mathrm{H}-3 \rightarrow \mathrm{L}+1(0.89)$ & $3.42(0.24)$ & $\mathrm{H}-3 \rightarrow \mathrm{L}+1(0.73)$ \\
\hline \multirow[t]{2}{*}{$\mathrm{Si}-\mathrm{Pc}^{\cdot}$} & $1.74(0.16)$ & $\mathrm{H} \rightarrow \mathrm{L}(0.89)$ & $2.59(1.04)$ & $\mathrm{S} \rightarrow \mathrm{L}+2(0.38)$ & $3.41(0.09)$ & $\mathrm{H}-3 \rightarrow \mathrm{S}(0.46)$ \\
\hline & $2.05(0.001)$ & $\mathrm{H} \rightarrow \mathrm{L}+1(0.97)$ & $2.76(0.22)$ & $\mathrm{S} \rightarrow \mathrm{L}+3(0.85)$ & $3.42(0.06)$ & $\mathrm{H}-3 \rightarrow \mathrm{S}(0.33)$ \\
\hline \multirow[t]{2}{*}{$\mathrm{Si}^{-\mathrm{Pc}^{+}}$} & $2.10(0.59)$ & $\mathrm{H} \rightarrow \mathrm{L}(0.92)$ & - & - & $3.47(0.00)$ & $\mathrm{H}-2 \rightarrow \mathrm{L}(0.71)$ \\
\hline & $2.11(0.59)$ & $\mathrm{H} \rightarrow \mathrm{L}+1(0.92)$ & & & $3.47(0.00)$ & $\mathrm{H}-2 \rightarrow \mathrm{L}+1(0.72)$ \\
\hline \multicolumn{7}{|c|}{$\omega \mathrm{B} 97 \mathrm{XD} / 6-311+\mathrm{G}(\mathrm{d}, \mathrm{p})$} \\
\hline Form & $\mathrm{E}_{Q}(\mathrm{eV})$ & MOs Configuration & $\mathrm{E}_{Q(S i)}(\mathrm{eV})$ & MOs Configuration & $\mathrm{E}_{B}(\mathrm{eV})$ & MOs Configuration \\
\hline \multirow[t]{2}{*}{$\mathrm{Si}-\mathrm{Pc}$} & $2.16(0.56)$ & $\mathrm{H} \rightarrow \mathrm{L}(0.89)$ & - & - & $3.39(0.24)$ & $\mathrm{H}-2 \rightarrow \mathrm{L}(0.78)$ \\
\hline & $2.16(0.56)$ & $\mathrm{H} \rightarrow \mathrm{L}+1(0.89)$ & & & $3.40(0.24)$ & $\mathrm{H}-2 \rightarrow \mathrm{L}+1(0.78)$ \\
\hline \multirow[t]{2}{*}{ Si-Pc } & $1.60(0.11)$ & $\mathrm{H} \rightarrow \mathrm{L}(0.92)$ & $2.54(0.44)$ & $\mathrm{S} \rightarrow \mathrm{L}+2(0.79)$ & $3.56(0.15)$ & $\mathrm{H}-6 \rightarrow \mathrm{L}(0.38)$ \\
\hline & $2.02(0.01)$ & $\mathrm{H} \rightarrow \mathrm{L}+1(0.97)$ & $2.56(0.74)$ & $\mathrm{H} \rightarrow \mathrm{L}(0.78)$ & $3.70(0.12)$ & $\mathrm{H}-2 \rightarrow \mathrm{S}(0.50)$ \\
\hline \multirow[t]{2}{*}{$\mathrm{Si}_{-} \mathrm{Pc}^{+}$} & $2.08(0.59)$ & $\mathrm{H} \rightarrow \mathrm{L}(0.93)$ & - & - & $3.58(0.00)$ & $\mathrm{H}-3 \rightarrow \mathrm{L}(0.51)$ \\
\hline & $2.09(0.58)$ & $\mathrm{H} \rightarrow \mathrm{L}+1(0.93)$ & & & $3.68(0.00)$ & $\mathrm{H}-4 \rightarrow \mathrm{L}+2(0.48)$ \\
\hline \multicolumn{7}{|c|}{$\omega \mathrm{B} 97 \mathrm{XD} / 6-311 \mathrm{G}(\mathrm{d})$ in Methanol Solution $(\mathrm{PCM})$} \\
\hline \multirow[t]{2}{*}{$\mathrm{Si}-\mathrm{Pc}$} & $2.06(0.76)$ & $\mathrm{H} \rightarrow \mathrm{L}(0.93)$ & - & - & $3.36(0.26)$ & $\mathrm{H}-3 \rightarrow \mathrm{L}(0.69)$ \\
\hline & $2.06(0.76)$ & $\mathrm{H} \rightarrow \mathrm{L}+1(0.92)$ & & & $3.37(0.26)$ & $\mathrm{H}-3 \rightarrow \mathrm{L}+1(0.69)$ \\
\hline \multirow[t]{2}{*}{$\mathrm{Si}-\mathrm{Pc}^{\cdot}$} & $1.57(0.17)$ & $\mathrm{H} \rightarrow \mathrm{L}(0.93)$ & $2.46(1.06)$ & $\mathrm{H} \rightarrow \mathrm{L}(0.78)$ & $3.44(0.01)$ & $\mathrm{H}-6 \rightarrow \mathrm{S}(0.43)$ \\
\hline & $2.26(0.01)$ & $\mathrm{H} \rightarrow \mathrm{L}+1(0.96)$ & $2.53(0.53)$ & $\mathrm{S} \rightarrow \mathrm{L}+2(0.82)$ & $3.45(0.03)$ & $\mathrm{S} \rightarrow \mathrm{L}+6(0.61)$ \\
\hline \multirow[t]{2}{*}{$\mathrm{Si}^{-\mathrm{Pc}^{+}}$} & $1.99(0.79)$ & $\mathrm{H} \rightarrow \mathrm{L}(0.92)$ & - & - & $3.70(0.00)$ & $\mathrm{H}-3 \rightarrow \mathrm{L}(0.74)$ \\
\hline & $2.00(0.78)$ & $\mathrm{H} \rightarrow \mathrm{L}+1(0.92)$ & & & $3.70(0.00)$ & $\mathrm{H}-4 \rightarrow \mathrm{L}(0.56)$ \\
\hline \multicolumn{7}{|c|}{$\overline{\text { B3LYP/6-311G(d) }}$} \\
\hline Form & $\mathrm{E}_{Q}(\mathrm{eV})$ & MOs Configuration & $\mathrm{E}_{Q(S i)}(\mathrm{eV})$ & MOs Configuration & $\mathrm{E}_{B}(\mathrm{eV})$ & MOs Configuration \\
\hline \multirow[t]{2}{*}{$\mathrm{Si}-\mathrm{Pc}$} & $2.21(0.49)$ & $\mathrm{H} \rightarrow \mathrm{L}(0.87)$ & $2.60(0.005)$ & $\mathrm{H}-1 \rightarrow \mathrm{L}(0.99)$ & $2.94(0.12)$ & $\mathrm{H}-3 \rightarrow \mathrm{L}(0.86)$ \\
\hline & $2.21(0.48)$ & $\mathrm{H} \rightarrow \mathrm{L}+1(0.87)$ & $2.61(0.004)$ & $\mathrm{H}-1 \rightarrow \mathrm{L}+1(0.99)$ & $2.95(0.12)$ & $\mathrm{H}-3 \rightarrow \mathrm{L}+1(0.86)$ \\
\hline \multirow[t]{5}{*}{$\mathrm{Si}-\mathrm{Pc}^{\prime}$} & $1.89(0.05)$ & $\mathrm{H} \rightarrow \mathrm{L}+1(0.44)$ & $2.25(0.32)$ & $\mathrm{S} \rightarrow \mathrm{L}+3(0.67)$ & $2.94(0.001)$ & $\mathrm{H}-1 \rightarrow \mathrm{L}(0.71)$ \\
\hline & $1.91(0.05)$ & $\mathrm{H} \rightarrow \mathrm{L}+1(0.25)$ & $2.36(0.49)$ & $\mathrm{S} \rightarrow \mathrm{L}+2(0.46)$ & $2.95(0.001)$ & $\mathrm{H}-1 \rightarrow \mathrm{L}(0.70)$ \\
\hline & & & $2.52(0.15)$ & $\mathrm{S} \rightarrow \mathrm{L}+4(0.83)$ & $3.03(0.01)$ & $\mathrm{H} \rightarrow \mathrm{L}+2(0.35)$ \\
\hline & & & & & $3.05(0.003)$ & $\mathrm{H}-3 \rightarrow \mathrm{L}(0.56)$ \\
\hline & & & & & $3.08(0.015)$ & $\mathrm{H}-3 \rightarrow \mathrm{L}(0.51)$ \\
\hline \multirow[t]{2}{*}{$\mathrm{Si}^{-\mathrm{Pc}^{+}}$} & $2.10(0.45)$ & $\mathrm{H}-1 \rightarrow \mathrm{L}+1(0.88)$ & - & - & $2.61(0.02)$ & $\mathrm{H}-2 \rightarrow \mathrm{L}(0.97)$ \\
\hline & $2.10(0.45)$ & $\mathrm{H}-1 \rightarrow \mathrm{L}+2(0.88)$ & & & $2.71(0.03)$ & $\mathrm{H}-3 \rightarrow \mathrm{L}(0.94)$ \\
\hline \multicolumn{7}{|c|}{ B3LYP/6-311+G(d,p) } \\
\hline Form & $\mathrm{E}_{Q}(\mathrm{eV})$ & MOs Configuration & $\mathrm{E}_{Q(S i)}(\mathrm{eV})$ & MOs Configuration & $\mathrm{E}_{B}(\mathrm{eV})$ & MOs Configuration \\
\hline \multirow[t]{2}{*}{$\mathrm{Si}-\mathrm{Pc}$} & $2.19(0.49)$ & $\mathrm{H} \rightarrow \mathrm{L}(0.86)$ & $2.53(0.00)$ & $\mathrm{H}-1 \rightarrow \mathrm{L}(0.99)$ & $2.91(0.12)$ & $\mathrm{H}-3 \rightarrow \mathrm{L}(0.87)$ \\
\hline & $2.19(0.49)$ & $\mathrm{H} \rightarrow \mathrm{L}+1(0.86)$ & $2.54(0.00)$ & $\mathrm{H}-1 \rightarrow \mathrm{L}+1(0.99)$ & $2.92(0.12)$ & $\mathrm{H}-3 \rightarrow \mathrm{L}+1(0.87)$ \\
\hline \multirow[t]{6}{*}{$\mathrm{Si}-\mathrm{Pc}$} & $1.87(0.05)$ & $\mathrm{H} \rightarrow \mathrm{L}+1(0.42)$ & $2.22(0.30)$ & $\mathrm{S} \rightarrow \mathrm{L}+3(0.67)$ & $2.92(0.0007)$ & $\mathrm{H}-1 \rightarrow \mathrm{S}(0.80)$ \\
\hline & $1.89(0.05)$ & $\mathrm{H} \rightarrow \mathrm{L}+1(0.27)$ & $2.33(0.41)$ & $\mathrm{S} \rightarrow \mathrm{L}+4(0.51)$ & $2.93(0.002)$ & $\mathrm{H}-1 \rightarrow \mathrm{L}(0.77)$ \\
\hline & & & $2.45(0.09)$ & $\mathrm{S} \rightarrow \mathrm{L}+5(0.82)$ & $2.95(0.0004)$ & $\mathrm{H}-2 \rightarrow \mathrm{S}(0.55)$ \\
\hline & & & $2.46(0.21)$ & $\mathrm{S} \rightarrow \mathrm{L}+4(0.82)$ & $2.95(0.0005)$ & $\mathrm{H}-1 \rightarrow \mathrm{L}+1(0.94)$ \\
\hline & & & $2.52(0.02)$ & $\mathrm{S} \rightarrow \mathrm{L}+5(0.43)$ & $3.02(0.007)$ & $\mathrm{H}-4 \rightarrow \mathrm{S}(0.43)$ \\
\hline & & & $2.57(0.09)$ & $\mathrm{S} \rightarrow \mathrm{L}+6(0.94)$ & $3.05(0.001)$ & $\mathrm{H}-1 \rightarrow \mathrm{L}(0.36)$ \\
\hline $\mathrm{Si}^{-\mathrm{Pc}^{+}}$ & $2.09(0.46)$ & $\mathrm{H} \rightarrow \mathrm{L}+1(0.89)$ & - & - & $2.65(0.02)$ & $\mathrm{H}-2 \rightarrow \mathrm{L}(0.97)$ \\
\hline & $2.09(0.46)$ & $\mathrm{H} \rightarrow \mathrm{L}+2(0.90)$ & & & $2.74(0.02)$ & $\mathrm{H}-3 \rightarrow \mathrm{L}(0.94)$ \\
\hline & & & & & $2.88(0.0001)$ & $\mathrm{H}-2 \rightarrow \mathrm{L}+2(0.78)$ \\
\hline & & & & & $2.93(0.002)$ & $\mathrm{H}-2 \rightarrow \mathrm{L}+1(0.68)$ \\
\hline & & & & & $2.95(0.001)$ & $\mathrm{H}-4 \rightarrow \mathrm{L}(0.86)$ \\
\hline & & & & & $2.96(0.0004)$ & $\mathrm{H}-8 \rightarrow \mathrm{L}(0.84)$ \\
\hline & & & & & $3.00(0.0006)$ & $\mathrm{H}-3 \rightarrow \mathrm{L}+1(0.84)$ \\
\hline & & & & & $3.06(0.0005)$ & $\mathrm{H}-7 \rightarrow \mathrm{L}(0.82)$ \\
\hline & & & & & $3.08(0.0001)$ & $\mathrm{H}-9 \rightarrow \mathrm{L}(0.91)$ \\
\hline
\end{tabular}


SI Table 2. The $Q$ band lowest excitations energies $(e V)$ of Si-Pc, Si-PhPc and Si-Pz at the B3LYP/6-311G(d) level.

\begin{tabular}{ccccc}
\hline Complex & $\mathrm{E}_{Q}(\mathrm{eV})$ & MOs Configuration & $\mathrm{E}_{B}(\mathrm{eV})$ & MOs Configuration \\
\hline Si-Pc & $2.21(0.49)$ & $\mathrm{H} \rightarrow \mathrm{L}(0.87)$ & $2.94(0.12)$ & $\mathrm{H}-3 \rightarrow \mathrm{L}(0.86)$ \\
& $2.21(0.48)$ & $\mathrm{H} \rightarrow \mathrm{L}+1(0.87)$ & $2.95(0.12)$ & $\mathrm{H}-3 \rightarrow \mathrm{L}+1(0.86)$ \\
Si-PhPc & $1.92(0.83)$ & $\mathrm{H} \rightarrow \mathrm{L}(0.91)$ & $2.75(0.00)$ & $\mathrm{H} \rightarrow \mathrm{L}+3(0.88)$ \\
& $1.92(0.83)$ & $\mathrm{H} \rightarrow \mathrm{L}+1(0.91)$ & $2.80(0.01)$ & $\mathrm{H}-1 \rightarrow \mathrm{L}+5(0.70)$ \\
Si-Pz & $2.28(0.00)$ & $\mathrm{H}-1 \rightarrow \mathrm{L}(0.83)$ & $2.38(0.03)$ & $\mathrm{H}-3 \rightarrow \mathrm{L}(0.73)$ \\
& $2.29(0.00)$ & $\mathrm{H}-1 \rightarrow \mathrm{L}+(0.83)$ & $2.39(0.03)$ & $\mathrm{H}-3 \rightarrow \mathrm{L}+1(0.73)$ \\
\hline \hline
\end{tabular}

SI Table 3. Activation energy and required $\mathrm{Si}-\mathrm{C}$ stretches from the fixed PESs.

\begin{tabular}{ccccc}
\hline Complex & \multicolumn{2}{c}{ B3LYP } & \multicolumn{2}{c}{$\omega$ B97XD } \\
& $\mathrm{d}_{S i-C}(\AA)$ & $\mathrm{E}_{a}(\mathrm{eV})$ & $\mathrm{d}_{S i-C}(\AA)$ & $\mathrm{E}_{a}(\mathrm{eV})$ \\
\hline $\mathrm{Si}-\mathrm{Pc}$ & 0.30 & 0.33 & 0.60 & 1.36 \\
$\mathrm{Si}-\mathrm{PhPc}$ & 0.55 & 0.98 & 0.75 & 1.96 \\
$\mathrm{Si}-\mathrm{Pz}$ & 0.05 & 0.05 & 0.20 & 0.19 \\
\hline \hline
\end{tabular}

SI Table 4. Activation energy and required $\mathrm{Si}-\mathrm{C}$ stretches from the relaxed PESs calculated with TDA-PCM (methanol). The Q and B band excitation energies $(\mathrm{eV})$ with their oscillator strengths provided in parentheses.

\begin{tabular}{lcccc}
\hline Complex & $\mathrm{d}_{\mathrm{Si}-\mathrm{C}}$ & $\mathrm{E}_{a}$ & $\mathrm{E}_{Q}$ & $\mathrm{E}_{B}$ \\
\hline \multicolumn{5}{c}{$\omega \mathrm{B} 97 \mathrm{XD} / 6-311 \mathrm{G}(\mathrm{d})$} \\
\hline $\mathrm{Si}-\mathrm{Pc}$ & 0.30 & 0.46 & $2.06(0.76)$ & $3.36(0.26)$ \\
$\mathrm{Si}-\mathrm{PhPc}$ & 0.50 & 0.94 & $1.83(1.13)$ & $3.46(0.42)$ \\
$\mathrm{Si}-\mathrm{Pz}$ & 0.05 & 0.01 & $2.40(0.22)$ & $3.12(0.24)$ \\
\hline
\end{tabular}




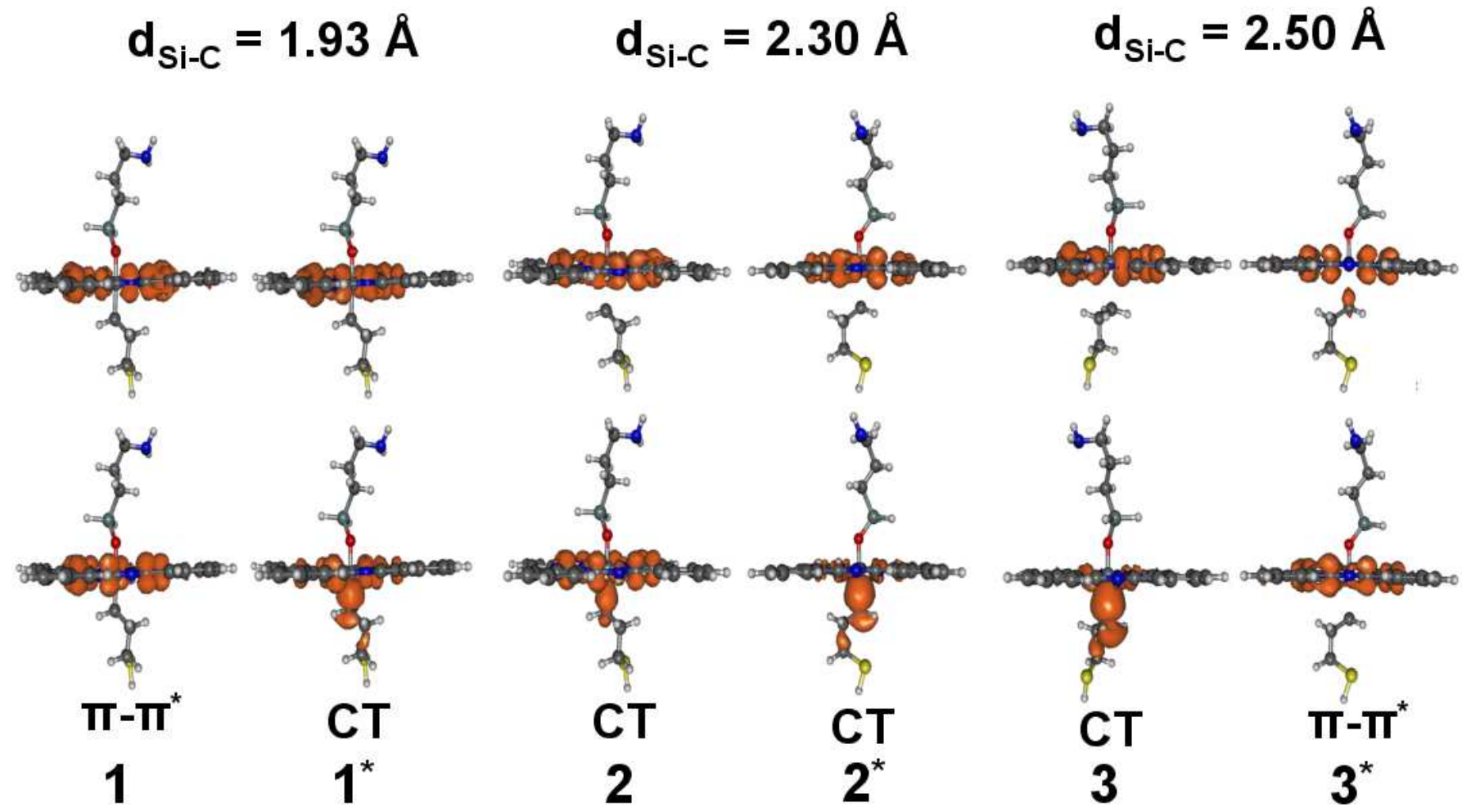

SI Figure 1. An increased reproduction of Figure 4 orbital pictures. 


\section{LUMO+1}

\section{LUMO}

\section{HOMO-3}
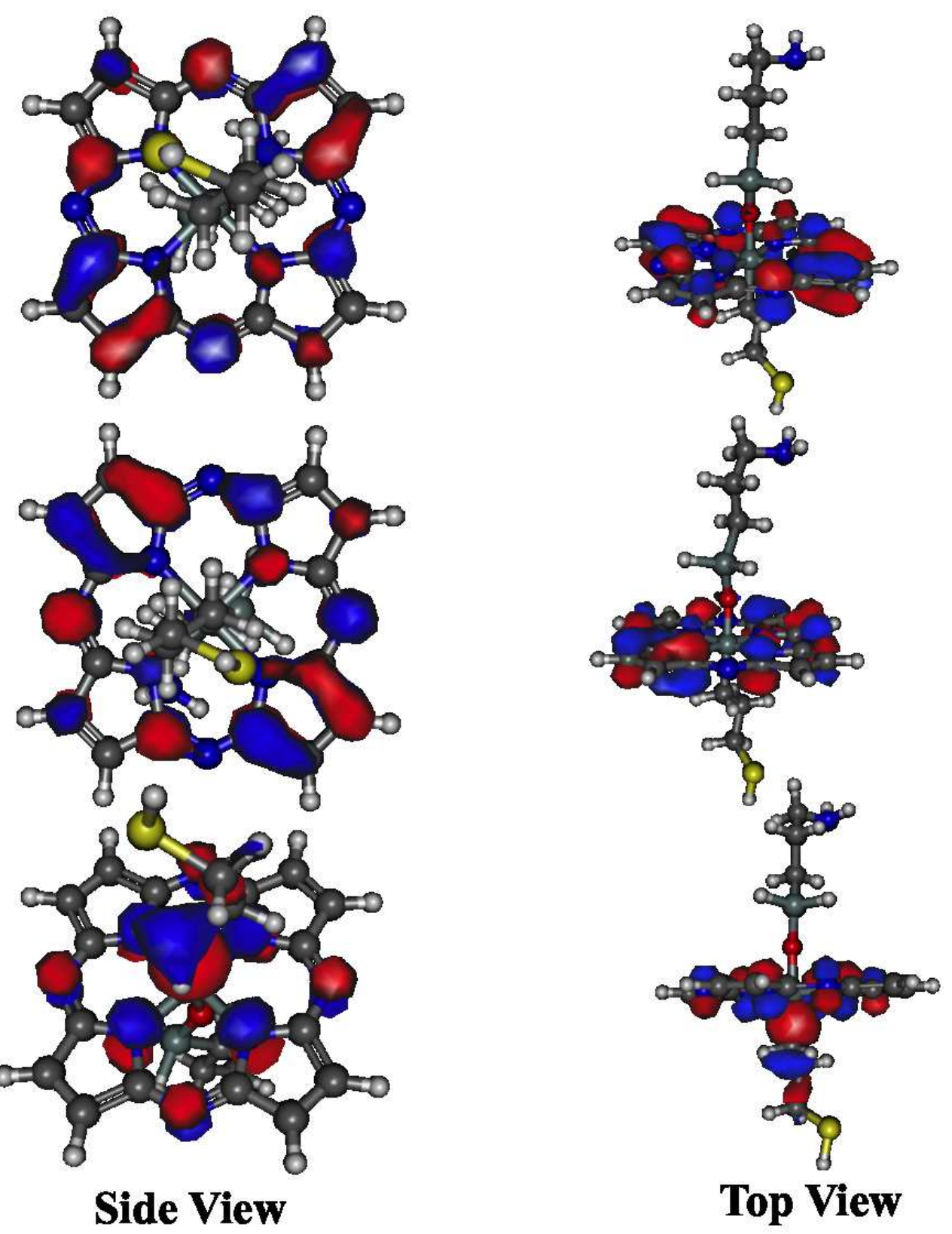

SI Figure 2. Isosurfaces of the orbitals involved in the charge transfer (CT) between core Si and Si-ligated atoms in Si-Pz complex. 

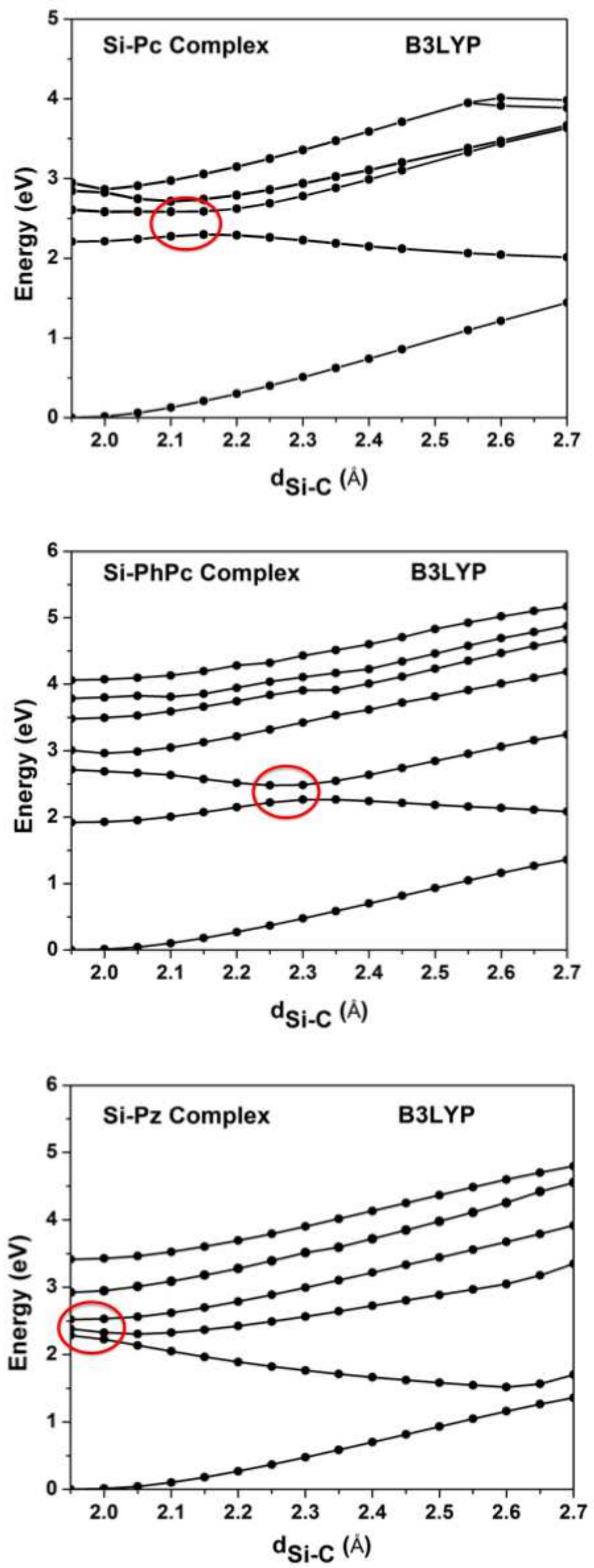

SI Figure 3. Relaxed PESs calculated at the B3LYP level. The red circle indicates the onset of repulsive nature in the lowest excited state. 

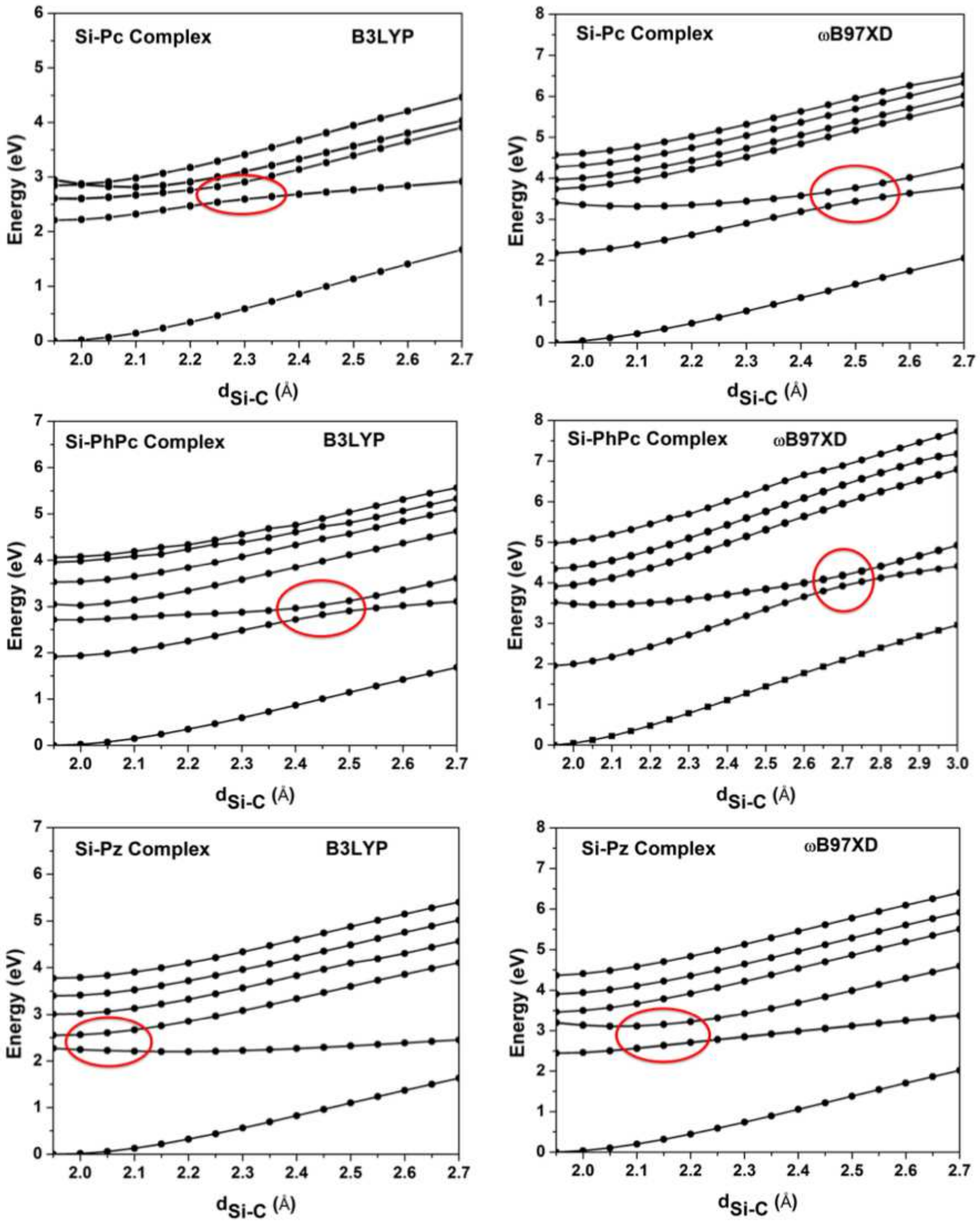

SI Figure 4. Fixed PESs calculated at levels of B3LYP and $\omega$ B97XD and 6-311G(d) basis set. The red circle indicates the onset of the repulsive nature in the lowest excited state. 

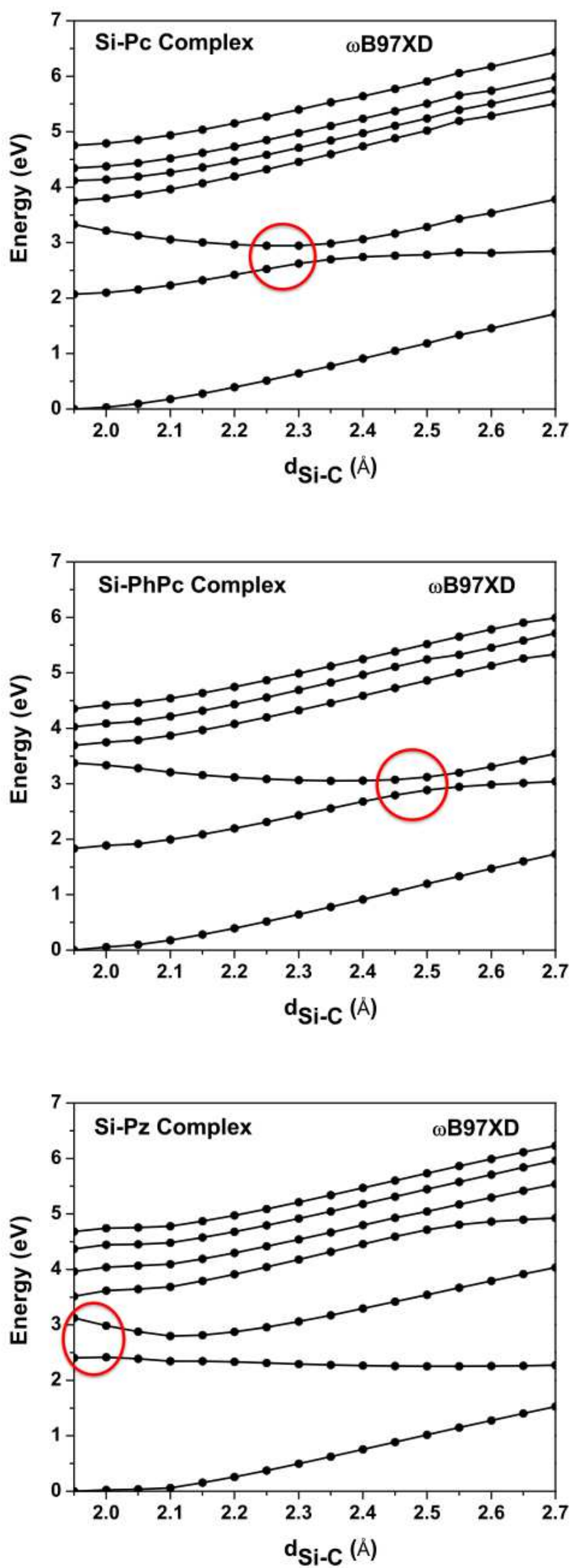

SI Figure 5. Relaxed PESs calculated at the $\omega$ B97XD level in methanol. The red circle indicates the onset of the repulsive nature in the lowest excited state. 
SI Table 5. The atomic coordinates of the optimized structures of Si-Pc complex using B3LYP functional and 6-31G(d) basis set in the gas phase and methanol solution. The unit of atomic position is in $\AA$. The root mean square deviation (RMSD) between the two structures is $0.06 \AA ̊$.

Gas Phase N $2.0545186996459961-0.3372983932495117-0.0616887025535107$ C $2.97777056694030760 .6538857817649841-0.2977137863636017$ C $4.27692747116088870 .0494933612644672-0.5480476021766663$ C $4.0963010787963867-1.3409051895141602-0.4509733021259308$ C $2.6905877590179443-1.5555384159088135-0.1440964490175247$ N - $0.1013273969292641-2.00666117668151860 .4655309021472931$ C $0.8809329271316528-2.95481204986572270 .2898512184619904$ C $0.2956674695014954-4.27987670898437500 .4256109595298767$ C - $1.0741013288497925-4.08846616744995120 .6758122444152832$ C - $1.2944155931472778-2.65030264854431150 .6884686350822449$ N - 1.74657082557678220 .20122881233692170 .6879023909568787 C -2.6833596229553223 -0.7943795919418335 0.8574219346046448 C -3.9988462924957275 -0.1940672695636749 1.0131008625030518 C -3.8185539245605469 1.19540190696716310 .9164797663688660 C -2.39643931388854981 .41451132297515870 .7041736245155334$ N 0.39990869164466861 .86699223518371580 .1801429688930511 C - 0.58737725019454962 .81669545173645020 .3009894192218781 C - 0.01171494834125044 .13808536529541020 .1035275012254715 C $1.35640406608581543 .9437177181243896-0.1488200426101685$ C $1.58691203594207762 .5079457759857178-0.1005270928144455$ N 2.7741000652313232 $1.9627707004547119-0.3109452426433563$ N $2.1628856658935547-2.75799918174743650 .0238347034901381$ N -2.4851481914520264 -2.1023578643798828 0.8799085021018982 N - 1.87261331081390382 .62003922462463380 .5478608012199402 C $0.8439784049987793-5.56048250198364260 .3421359360218048$ C $-1.9360241889953613-5.17183256149291990 .8516435027122498$ C $-1.3885586261749268-6.45088958740234380 .7709083557128906$ C $-0.0158548541367054-6.64275360107421880 .5193222761154175$ H - $2.9928333759307861-5.01475954055786131 .0427600145339966$ H -2.0295174121856689 - 7.31830263137817380 .9027367830276489 H $0.3762961924076080-7.65449857711791990 .4626677334308624$ H $1.9024908542633057-5.69863796234130860 .1459794342517853$ C $5.52662467956542970 .5989941358566284-0.8365594744682312$ C $5.1609091758728027-2.2234525680541992-0.6384614706039429$ C $6.5902714729309082-0.2818292677402496-1.0228065252304077$ C $6.4098243713378906-1.6754336357116699-0.9246569275856018$ H $5.0121350288391113-3.2958352565765381-0.5619725584983826$ H $7.2621788978576660-2.3321213722229004-1.0756922960281372$ H $7.57838869094848630 .1101559177041054-1.2475435733795166$ H $5.65593576431274411 .6740725040435791-0.9100869297981262$ C $2.20885968208312995 .0220885276794434-0.3873977065086365$ C -0.5696836709976196 5.4166626930236816 0.1270476430654526 C $0.28146812319755556 .4948668479919434-0.1082544028759003$ C $1.65311336517333986 .2999124526977539-0.3622209429740906$ H $3.26432967185974124 .8623366355895996-0.5829876065254211$ H $2.28693485260009777 .1641597747802734-0.5409522056579590$ H - $0.11692078411579137 .5056672096252441-0.0965177342295647$ $\mathrm{H}-1.62795877456665045 .55643987655639650 .3229165673255920$ C -4.89794635772705082 .07441401481628421 .0160917043685913$ C -5.2636919021606445 -0.7481535077095032 1.2143507003784180 C -6.3417572975158691 0.12865839898586271 .3170301914215088 C -6.1612277030944824 1.52241444587707521 .2187052965164185 $\mathrm{H}-4.74927330017089843 .14653396606445310 .9365293383598328$ H -7.0253028869628906 2.1758785247802734 1.3015567064285278 H -7.3417782783508301 -0.2663724422454834 1.4734009504318237 H -5.3930182456970215 -1.8234318494796753 1.2850896120071411 Si $0.1572316884994507-0.06828743219375610 .3613847792148590$ C $0.6704255938529968-0.01861653104424482 .2458858489990234$ H $1.3280415534973145-0.88510966300964362 .4126014709472656$ H 1.31332015991210940 .86048763990402222 .3937549591064453
Methanol Solution

N -2.2227694988250732 $0.0958619043231010-0.3859835565090179$ C -3.0145626068115234 1.1897386312484741 -0.6491810083389282 C $-4.40333604812622070 .7667775154113770-0.7466384768486023$ C -4.4125294685363770 - $0.6230796575546265-0.5305053591728210$ C -3.0295038223266602 -1.0157277584075928 -0.3080652356147766 N - $0.2863022983074188-1.82468032836914060 .1496412456035614$ C - $1.4051929712295532-2.62780904769897460 .1415504664182663$ C - $1.0139344930648804-3.99901580810546880 .4311425983905792$ C $0.3786453008651733-3.98468518257141110 .6258646249771118$ C $0.8048709034919739-2.60454463958740230 .4485846459865570$ N 1.65666294097900390 .13597945868968960 .1313658505678177 C $2.4412589073181152-0.94987607002258300 .4523298442363739$ C $3.8146834373474121-0.51183021068573000 .6477566957473755$ C 3.82353711128234860 .87824410200119020 .4398313760757446 C 2.45542049407958981 .25800740718841550 .1250138878822327 N - $0.26402717828750612 .0514507293701172-0.4178026318550110$ C $0.84178936481475832 .8663110733032227-0.3555997014045715$ C 0.44178655743598944 .2440481185913086 -0.5952630043029785 C $-0.94937318563461304 .2257404327392578-0.7986177206039429$ C - $1.36630952358245852 .8371882438659668-0.6765881776809692$ N -2.6276075839996338 2.4494547843933105 -0.7927759885787964 N -2.6610004901885986 -2.2672493457794189-0.0752914622426033 N 2.0636670589447021 -2.2124276161193848 0.5834036469459534 N 2.0945107936859131 2.5095047950744629 -0.1127211749553680 C - $1.7479029893875122-5.18223571777343750 .5353553295135498$ C $1.0793219804763794-5.15351343154907230 .9304203987121582$ C $0.3470617234706879-6.33638525009155271 .0323624610900879$ C - $1.0483430624008179-6.35046148300170900 .8370859026908875$ H $2.1541299819946289-5.14027070999145511 .0825161933898926$ H $0.8594608306884766-7.26508760452270511 .2666101455688477$ $\mathrm{H}-1.5868327617645264-7.28980684280395510 .9244273304939270$ $\mathrm{H}-2.8230414390563965-5.19086265563964840 .3854626119136810$ C $-5.58519506454467771 .4692326784133911-0.9912536144256592$ C $-5.6034526824951172-1.3518373966217041-0.5531938076019287$ C -6.7749147415161133 $0.7415931820869446-1.0147927999496460$ C $-6.7838068008422852-0.6508246660232544-0.7984060049057007$ H $-5.6085214614868164-2.4243228435516357-0.3851046264171600$ H -7.7289724349975586 -1.1855903863906860 -0.8231648802757263 $\mathrm{H}-7.71335697174072271 .2553641796112061-1.2026876211166382$ H -5.5764851570129395 2.5420372486114502 -1.1571729183197021 C - $1.65947818756103525 .4013023376464844-1.0516331195831299$ C $1.16528892517089845 .4375700950622559-0.6393049359321594$ C $0.45692557096481326 .6115369796752930-0.8937364220619202$ C - $0.93733668327331546 .5936932563781738-1.0975440740585327$ $\mathrm{H}-2.73374152183532715 .3857436180114746-1.2071597576141357$ $\mathrm{H}-1.45694673061370857 .5273733139038086-1.2929470539093018$ H $0.98714321851730357 .5586538314819336-0.9355536699295044$ H 2.2390489578247070 5.4494414329528809 -0.4800255000591278 C 5.00004386901855471 .62145268917083740 .5560356974601746 C $4.9829082489013672-1.20206010341644290 .9786085486412048$ C $6.1588854789733887-0.46106079220771791 .0929564237594604$ C 6.16729784011840820 .93268245458602910 .8841581344604492 H 5.00384712219238282 .69509339332580570 .3958101570606232 H 7.10155439376831051 .47813618183135990 .9821684956550598 H $7.0868372917175293-0.96480065584182741 .3479336500167847$ H $4.9738779067993164-2.27533030509948731 .1412622928619385$ Si $-0.27039071917533870 .1048574075102806-0.1784007102251053$ C $-0.1512441784143448-0.1868466287851334-2.1023583412170410$ H - $0.7844434380531311-1.0576840639114380-2.3304321765899658$ H -0.6519355773925781 $0.6673614978790283-2.5809493064880371$ 
C -0.4114517569541931 -0.0379205271601677 3.3386747837066650 H -1.0648578405380249 0.83982753753662113 .2601597309112549 H - $1.0585039854049683-0.91596114635467533 .2053120136260986$ C $0.1340105533599854-0.11187923699617394 .7711210250854492$ H - $0.6907501220703125-0.20953534543514255 .4838151931762695$ H $0.7952184677124023-0.97789639234542854 .8816242218017578$ S 1.08334684371948241 .42262589931488045 .1989989280700684 H 1.20548367500305181 .16258215904235846 .5178542137145996 O - $0.2304037660360336-0.1704740375280380-1.3114596605300903$ Si $0.16284790635108950 .1062665209174156-2.8812406063079834$ H $1.3513317108154297-0.7088647484779358-3.2895750999450684$ H $0.54080218076705931 .5401945114135742-3.0902779102325439$ C - $1.2890775203704834-0.3286466300487518-3.9998743534088135$ H - $1.5783102512359619-1.3717781305313110-3.8052306175231934$ H -2.1598131656646729 $0.2812058627605438-3.7299854755401611$ C -0.9887194633483887 -0.1454778611660004 -5.4977254867553711 H $-0.1710584014654160-0.8110388517379761-5.8068351745605469$ $\mathrm{H}-0.63110017776489260 .8785973787307739-5.6885170936584473$ C -2.1960029602050781 -0.4136905074119568 -6.4070940017700195 H - $1.8581786155700684-0.4016392529010773-7.4601955413818359$ H - $2.5752658843994141-1.4247944355010986-6.2097582817077637$ $\mathrm{N}-3.28575038909912110 .5298691391944885-6.1275739669799805$ H -3.0068101882934570 $1.4650946855545044-6.4234485626220703$ H $-4.10380411148071290 .2904156148433685-6.6851038932800293$
C $1.2135993242263794-0.4105863869190216-2.7710738182067871$ H $1.86874008178710940 .4569593667984009-2.6214632987976074$ H $1.7207016944885254-1.2659729719161987-2.3043291568756104$ C $1.1477792263031006-0.7236553430557251-4.2708606719970703$ H $2.1477134227752686-0.9318213462829590-4.6608371734619141$ H $0.5120822191238403-1.5949023962020874-4.4561576843261719$ S $0.46361929178237920 .7148872017860413-5.2289400100708008$ Н $0.81294596195220950 .2758340537548065-6.4551734924316406$ O -0.4682203531265259 0.32068964838981631 .5275623798370361 Si - 1.23388504981994631 .10373032093048102 .7531781196594238 H -2.6706449985504150 0.68430227041244512 .8411834239959717 H - 1.24684345722198492 .58469986915588382 .5226523876190186 C -0.3778156042098999 0.73615676164627084 .3889670372009277 H - $0.3222678601741791-0.35471713542938234 .5186395645141602$ Н 0.65938657522201541 .09052419662475594 .3325681686401367 C - 1.07522106170654301 .36831223964691165 .6071329116821289 H -2.0962557792663574 0.97413051128387455 .7003374099731445 H - 1.18045985698699952 .45430397987365725 .4618625640869141 C -0.3520346879959106 1.12611770629882816 .9390635490417480 H -1.0040428638458252 1.47141659259796147 .7603397369384766 H -0.2100795954465866 0.04670748487114917 .0815806388854980 N 0.97807103395462041 .75976455211639406 .9567022323608398 H 0.85160619020462042 .77295279502868656 .9658045768737793 H 1.43241906166076661 .54321289062500007 .8438868522644043 
SI Table 6. The atomic coordinates of the optimized structures of Si-PhPc complex using B3LYP functional and 6-31G(d) basis set in the gas phase and methanol solution. The unit of atomic position is in $\AA$. The root mean square deviation (RMSD) between the two structures is $0.09 \AA$.

\begin{tabular}{|c|c|}
\hline Gas Phase & Methanol Solution \\
\hline N $1.6329030458591118-1.23847612749868330 .2729644750408728$ & N $1.6149536618904345-1.22122668966903360 .2373370967123394$ \\
\hline C $2.9356621385994770-0.80173392588247380 .1941350612191298$ & C $2.9104154942990963-0.76516670988449000 .1394466587867991$ \\
\hline C $3.8206332219498975-1.95158068632517260 .0938021343195978$ & C $3.8127638371658064-1.90010297117161890 .0287897775965794$ \\
\hline C $2.9861178934999550-3.10174144842593020 .1160970247648112$ & C $2.9963093153227010-3.06423834660703020 .0669640055086403$ \\
\hline C $1.6201598607349303-2.61364319519072950 .2293675962328877$ & C $1.6254711623186888-2.59638856968458360 .2006984886809034$ \\
\hline N -1.1116541555368413 -1.6678897778898789 0.3953451296752353 & N -1.1202546978949699 -1.6937846656699886 0.4111526818008662 \\
\hline C - $0.6757924878639783-2.97369041950738880 .3442125766290405$ & C -0.6648321374348455 -2.9939543859364077 0.3680106344072328 \\
\hline C -1.8270472170620653 -3.8629806891251586 0.3622370549778612 & C -1.7985180436422725 -3.9028798551694464 0.4332791177152289 \\
\hline C -2.9762953139760180 -3.0290728562298419 0.4137585838948715 & C -2.9610335466643654 -3.08763374899689240.5054239592119135 \\
\hline C -2.4867213881623891 -1.6593435723116639 0.4260188446308045 & C -2.4941994831381589 - 1.71021766879393010 .4824280065529377 \\
\hline N -1.5408556729698875 1.07132817946551940 .4122154794434482 & N -1.5942217502919684 1.03693415589409650 .4217801687254977 \\
\hline C -2.8479537480030235 0.63657697420074830 .4161768845713715 & C -2.8940630324674688 0.58282684047071120 .4601748507327881 \\
\hline C -3.7365989304586562 1.78676082335673340 .3680229621641322 & C -3.8032545495983334 1.71685837508472550 .4249779532333871 \\
\hline C -2.9021618200520769 2.9352718941332792 0.3243411 & C -2.9889488126922710 2.87971230453482900 .355551130 \\
\hline C - 1.53227387607269332 .44767939220027530 .347 & C -1.6113837993283233 2.4139136239936234 0.3511245186931891 \\
\hline N 1.19635371803111661 .51186945835251700 .31160398018 & N 1.13423238523057761 .52228218036328380 .2721395213284543 \\
\hline C 0.76250862571589062 .81683555396681260 .2 & C 0.67961863899735092 .82013867765227030 .2425251101641061 \\
\hline C 1.91186923284201043 .70350686006849910 .190 & 8401897482360.1379 \\
\hline C 3.06027054404636802 .867402167375 & 7262.909 \\
\hline C 2.57253573706443911 .49868142495564680 .2 & C 2.50955562079901951 .53325108456508460 .1764170501957478 \\
\hline N 3.37424896841997010 .44722769476367290 .1 & 76932230.49245088212034310 .126 \\
\hline N $0.5690975759329774-3.41577373491813410 .27$ & N $0.5864913319594771-3.2$ \\
\hline N -3.2909350967990885 -0.6089588062479835 0.4476036898648450 & N $-3.3146029132270338-0.6$ \\
\hline 84423.255286287752 & $\mathrm{~N}-0$. \\
\hline $827-5.2353$ & 3633609105 \\
\hline C -4.2476490223894903 -3.5575836339949367 0. & C -4.2225267460599172 -3.6373922040004056 0.577 \\
\hline C -4.3977178534436039 -4.9673857802029930 0.4 & C -4.3465942268298665 -5.05096378613867000 .585 \\
\hline C -3.2282673406546292 -5.81568770406307320.3 & $9466-5.879655834$ \\
\hline $\mathrm{H}-5.1$ & $\mathrm{H}-5$. \\
\hline H -1.0500915913348221 -5.864441637 & 154690.379044072 \\
\hline C $5.1883077010442733-2.0563625693650778-0.0$ & $8-1.98341296430$ \\
\hline C 3.5 & 792014 \\
\hline C $5.7638201718245163-3.3484444158522555-0.1221571045293252$ & C $5.7749044839079620-3.2678762461170296-0.2115396114174113$ \\
\hline $1-4.51864$ & C 4.9 \\
\hline $009208-5.245697284$ & $1-5.2164657928990$ \\
\hline Н $5.8169494438708496-1.1707705513902815-0$. & $6-1.0929627885861$ \\
\hline C 4.32 & 356570 \\
\hline C 2.01752019399224075 .07423064633423240 .11 & 51953915.09995789984 \\
\hline 7535.6508230027 & 53776 \\
\hline 385377274.80062819779 & C 4.35729909080759734 .86699994914541 \\
\hline 80084322.745946678 & 2522.83142525836127 \\
\hline H 1.13327367329961405 .70486209080795170 .13703 & H 1.00298097747795925 .72264942469187510 .0877739157123601 \\
\hline C -3.4297393615039762 4.2058328708097488 0.2624 & C -3.5395961757943049 4.1419188124320545 0.2993847604815451 \\
\hline C -5.10930121517442881 .893399233 & C -5.17862922219146601 .7 \\
\hline C -5.68866379940456123 .1858860424 & C -5.7808298003968366 3.0825124070728682 0.3801256330219729 \\
\hline C -4.83945926360598484 .3551065661 & 592090229171460.3115 \\
\hline H -2.7839063662453811 5.0 & H -2.9172948452423757 5.03066020481083060 .2445131169571094 \\
\hline H -5.7391969539407066 1.00889250206360390 .3761626207 & H -5.7994333309345203 $0.9089898874250536 \quad 0.4871027123613663$ \\
\hline Si $0.0456188782913724-0.07971035044055180 .39$ & Si $0.0101828922778985-0.08780818966830880 .3868157068665286$ \\
\hline O -0.0644388286311673 -0.0851173020866645 -1.3163975398686973 & O -0.1308451295489023 -0.1001607845085128 -1.3354517601748566 \\
\hline Si 0.56514124565759820 .05084304 & $0650.0593078443731634-2.8026715970149052$ \\
\hline H $1.6384339254686553-0.9707058453568790-3.0447481793765299$ & H $1.6682489572140957-0.9683347736873595-2.9824464693707262$ \\
\hline H $1.20383382997295031 .3917230460481216-3.0187745430574724$ & Н $1.26223298290032141 .3939783227646629-2.9291345351260110$ \\
\hline C -0.7963738449706835 -0.1763180098835179 -4.1057778964586271 & C -0.6890263978826691 -0.1237105727030952 -4.1688084029571986 \\
\hline H -1.3102892159403179 -1.1273473652771169 -3.9033371968012895 & H -1.2048478108980394 -1.0866605338687652 -4.0394578840127613 \\
\hline H -1.5497628459105648 $0.6096135422553491-3.9717289634162145$ & H -1.4540488037179273 $0.6525863607462985-4.0404384116541818$ \\
\hline C -0.2992928626688187 $-0.1534900882272570-5.5612047120038994$ & C -0.1000242661671399 -0.0377962289846009 -5.5887440172408960 \\
\hline Н $0.3695617615502068-1.0042893904720909-5.7487430924$ ? & Н $0.6254484407800782-0.8478184037704495-5.7433628735756717$ \\
\hline Н $0.30450753621650860 .7498482522066218-5.7423069214398659$ & Н $0.46100398877073330 .9014040464033705-5.7094810893387953$ \\
\hline C -1.4317878465049285 -0.1925644126181294 -6.5964514480560270 & 6.7040354096898032 \\
\hline
\end{tabular}


$\mathrm{H}-0.9921197026738361-0.3261116057454327-7.6024373871183002$ H -2.0591053528009242 - $1.0736045986534228-6.4074203175111766$ $\mathrm{N}-2.28982364551220250 .9932467813536616-6.4811960119826804$ H - $1.77229580893508111 .8173641553641422-6.7860643100977258$ H -3.0855813807487409 $0.9107214133884359-7.1115272831183152$ C -7.0995181345663765 3.3682839086150200 0.2565451836045732 C -7.65255778425559634.62566436411939820.1910626336400143 C -6.8193751249198149 5.7729578763385607 0.1505014522391608 C -5.45099460348573755 .63827871168880620 .1756178481377156$ $\mathrm{H}-7.73605052308270922 .48728352116291560 .2870494888291965$ H -8.7323007599262734 4.74507872969625310 .1696729453808372 H -7.2681342972469869 6.76115033211545930 .0989686724124799 $\mathrm{H}-4.81053002383291876 .51649524614257380 .1436362842579793$ C -3.4115420362008959 - 7.22645872960420640 .3331133615702878 C -4.6700828391917728 - 7.78041286510558990 .3561820990461763 C $-5.8175704656892266-6.94809243936089340 .4053824451247375$ C -5.6819532960824013 -5.5797349556154305 0.4310705715203938 H -2.5303248549891002 -7.8623018905061830 0.2951283465850009 H - $4.7901179867319428-8.86010617866954320 .3364391868489885$ H $-6.8067503600998345-7.39731064738237090 .4228563268362739$ H -6.5605175031143066 -4.9400308782893356 0.4690064574075701 C $7.7179049574837126-4.7849358736949226-0.3565744558957140$ C $6.8858847780635157-5.9333498447499853-0.3338345819140190$ C $5.5227602982494419-5.8009224540645201-0.2072261617950616$ C $7.1689982631407014-3.5282370880798317-0.2523263010832338$ H $8.7933054424937342-4.9028989329823984-0.4575366724355258$ H $7.3315061968540371-6.9208575862064832-0.4167856526487509$ H $4.8833390980934723-6.6803021331818915-0.1897362589832884$ H $7.8043064128218260-2.6460688721673535-0.2702111696605868$ C $5.75932094398743385 .4088819219728741-0.1156842982103967$ C $3.48988598355490747 .0593658365566085-0.0662736991780228$ C $4.74596454131729227 .6094658113842408-0.1718274017586020$ C $5.89280565203212356 .7753514241774146-0.1965920765562937$ H $6.63732236437068404 .7676599102250998-0.1347473403006049$ H 2.6090692590504645 7.6965657472328699 -0.0472760650943782 H $4.86457321874849858 .6875602415024229-0.2369574382534782$ H $6.87988087792339187 .2218681194118757-0.2796382547153944$ C $0.2447786545083764-0.14487819277897172 .3355895905696786$ H $0.0951783053969248-1.18625264434395232 .6594095695171132$ H 1.30434798994474410 .06716155787196512 .5367500638507621 C -0.6091740814606339 0.76586674046748703 .2309196184497067 H - 0.44087174530495381 .82074731929807682 .9779600326657945 H - 1.67628378515740860 .57319142465126343 .0573674705518967 C -0.37704554458207170 .58322304478767864 .7359303656189491$ H - 1.05929868992347381 .22624215436400305 .3004570451182591 H -0.5604933946717297 -0.4568131014968373 5.0262981179856974 S 1.35888332509178781 .03591064672060615 .2090939225808217 H 1.14785862900945481 .06613223358446906 .5417961457616371
H -0.6309102047599318 -0.2143054550978644 -7.6734033954148613 H - $1.7385094042525466-1.0421818056462846-6.5728724714729543$ N -2.0843244177515068 $1.0151376823492062-6.6571699687041450$ H $-1.57701257850783501 .8594271283875352-6.9262151950694761$ H -2.7904949658827194 $0.8900987618340966-7.3824952567911675$ C -7.19564647578266843 .23788779605353620 .3866104228594595$ C -7.7706987726244803 4.4872279444577678 0.3284012131885355 C -6.9580407566314344 5.6491531591750093 0.2617173497368071 C -5.5858166482961717 5.5397740222625664 0.2537462628194453 H - 7.8149334141338818 2.3458954934671881 0.4379230378637867 $\mathrm{H}-8.85231954311652564 .58901597376882900 .3333502132659290$ H -7.4257347829235210 6.62869910524548800 .2162159206979877 H -4.9610831927288626 6.42804803951304040 .2017408343388929 C $-3.3192932900785102-7.29436292434842580 .5272507099960325$ C - $4.5679032859960387-7.86867153311619830 .6066636575297197$ C $-5.7290071308615724-7.05523779363049730 .6755867740106980$ C -5.6196153522421959 -5.6829340269365574 0.6646091531135502 $\mathrm{H}-2.4278720440750741-7.91432053063733760 .4740886482769716$ $\mathrm{H}-4.6696059630896354-8.95027082662363500 .6166991687690274$ H -6.7079771715239946 -7.5222535513127236 0.7376328395062428 H -6.5073294838594125 -5.05753579425161700 .7182044369918401$ C $7.7475464239348462-4.6737153735222057-0.4700396353722195$ C $6.9335041425642716-5.8359126295981740-0.4321406190533865$ C $5.5690194111017828-5.7262281014853693-0.2860386237467851$ C $7.1816579300534071-3.4235219207289367-0.3615020539803576$ H $8.8229029260248417-4.7761577811707969-0.5861172027196944$ H $7.3943180982439358-6.8159495631715661-0.5191148530624720$ H $4.9431614032859716-6.6147196030243336-0.2565666264855218$ H $7.8018756768660218-2.5312343953489189-0.3911910425854817$ C $5.62908375399002115 .4948087262055196-0.2128867898992599$ C $3.32781475127096647 .1096581921816311-0.1433588601016298$ C $4.57504761296567347 .6797106247947582-0.2633354095176400$ C $5.73660940324882956 .8645550946926583-0.2982484976035663$ H $6.51695767844399584 .8679622936662490-0.2400784130473783$ H 2.4361438797216000 $7.7309305184973631-0.1166275703666571$ H $4.67559379323153398 .7592918889635563-0.3321587434045368$ H $6.71437356954239297 .3286182230286423-0.3930455236941230$ C $0.2457137681342053-0.14155788144597382 .3213756507044585$ H $0.1192244289058055-1.18460045348177892 .6489430633839466$ H 1.30583793472290850 .09160914139246972 .4976593334067156 C - 0.60789725583496860 .75735738403028553 .2290189262133540 H - 0.45642446538412711 .81447543604651982 .9741252866437069 H - 1.67419773541465130 .55095060571023743 .0705533533826035 C -0.3592254673846093 0.5727177372430413 4.7299517539423812 $\mathrm{H}-1.02751019051228211 .21618264792349385 .3084206274620209$ H - $0.5346315056675539-0.46651286124716555 .0247000088158948$ S 1.38490773754247561 .02345034772383955 .1928258934677052 H $1.1873864464863868 \quad 1.08991111250977806 .5249781594651530$ 
SI Table 7. The atomic coordinates of the optimized structures of Si-Pz complex using B3LYP functional and 6-31G(d) basis set in the gas phase and methanol solution. The unit of atomic position is in $\AA$. The root mean square deviation (RMSD) between the two structures is $0.32 \AA$.

Gas Phase

N 0.59394562597233952 .09861186133022490 .1784123570994108 C 0.64900499234441062 .65301456943469831 .4334050458530405 C 0.77201046541522944 .09578421083774561 .3173679856271170 C $0.78986131043130494 .3882821406576582-0.0085053464932102$ C $0.68004910328217463 .1289175053378697-0.7240443226463836$ N $0.46646318871108380 .6309200412695471-2.1272495736299870$ C $0.55561410162347981 .8844454055154289-2.6824062629162770$ C $0.49331309766741991 .7754477039152414-4.1301123227508683$ C $0.35402640952693900 .4575119429753515-4.4239610898581700$ C $0.3301814688991866-0.2595942125039879-3.1603172388353551$ N $0.2325662670634958-1.6564747479146715-0.6566556455079942$ C $0.1114289207922984-2.2030098612044702-1.9099437342710539$ C $-0.1282500870045659-3.6311298350698857-1.7932939270341579$ C - $0.1666838242632129-3.9209315113690710-0.4679717405862430$ C $0.0507881536732716-2.67491228265427950 .2464774761749804$ N $0.3774971967853022-0.20019179194804331 .6483660230328978$ C $0.1979636027054307-1.43941745297254762 .2066376953658478$ C $0.1725418921113327-1.31897652457045593 .6549208505965809$ C $0.3318715653703792-0.00313414289296233 .9465040715339872$ C 0.45241199091426120 .70223369849775042 .6817269349589998 N 0.59294469261802252 .01973522271736042 .5997149714474688 N $0.66904891684749313 .0459578790890478-2.0496615569883407$ N $0.1776611208530550-1.5767044158996504-3.0786735341692326$ N $0.0517196502035222-2.59696936152345131 .5716670000955240$ Si $0.46543036755244190 .2116551844977233-0.2393488374090788$ C $2.42173895563965800 .1473264637152447-0.1886243479936645$ H $2.80399543776888780 .8144256598857782-0.9770226052706488$ H 2.72424856535812410 .62209459120488520 .7554045649804405 C $3.1506781074513932-1.1999498288455306-0.3045871981383295$ H $2.8099842497388750-1.88954042202566110 .4788049055732955$ H $2.9082903183458617-1.6803052413741315-1.2624171677337004$ C $4.6814709845854958-1.1101795596944501-0.2413528569824012$ H $5.1227088405083574-2.0976319788940776-0.4090328090205281$ H $5.0551208934465777-0.4275804664266287-1.0119656225240172$ S 5.2477631012345167 -0.5058875267779089 1.4185632012982827 H $6.5545534734849173-0.79708755989189491 .2479890761801891$ O - $1.25892574623684950 .3331383704069124-0.2674589030722487$ Si -2.6953774587393156 1.04373631007921320 .0641034423317611 H - $2.96730284887676772 .1650864091575182-0.8923109493678921$ H -2.7034536425575011 1.64711409752218721 .4362452442823790 C -4.0850672315713146 -0.2245586999294166 -0.0515238231194974 H -4.0774425220003829 -0.6629321954453853 - 1.0604931347847244 H -3.8734244346913713 - 1.05214733047696110 .6369082301078706 C -5.48263332840851620 .33917402853565630 .2545822276390934$ $\mathrm{H}-5.75318599650269751 .1108867175692150-0.4792623210685831$ H -5.4811705600837124 0.84196309832179531 .2341940356298311 C -6.5878333547311474 -0.7263551225540210 0.2582779536836164 H -7.5694170114262294 -0.2221860073432107 0.3318363771967472 H -6.5773108396042055 - $1.2558492454568380-0.7033747496423111$ N -6.3560402739761530 - 1.72163238631928791 .3126859870476393 H -6.4940369406202283 - 1.28720293031206892 .2249049133283330 H -7.0483052903506920 -2.4659607922112334 1.2478594991325787 H 0.82940543092092104 .75929889410299102 .1688194165484327 H $0.86592023026110005 .3483017610858603-0.4996319947446607$ H $0.54365323084946472 .6270060844816365-4.7941788599968724$ H $0.2652090515138871-0.0274416481200985-5.3861036436980134$ H - $0.2540977244402572-4.2847596753098678-2.6450825980117303$ H -0.3316091877099386 -4.86835269748001130.0258470148740849 H $0.0449276793715201-2.16094627445053924 .3207398417313296$ H 0.36276438769271550 .48977093360277924 .9080551690377856
Methanol Solution

N 2.0229043236973809 -0.3379236903844109 -0.0699784621649242 C $2.95362938974319000 .6381270372565169-0.3143617020078151$ C $4.24326618986152580 .0229304393624895-0.5706033338884662$ C $4.0693246423413409-1.3178827831440141-0.4734823257776357$ C $2.6708831514610307-1.5436506495556042-0.1560393319380060$ N - $0.0953008093904783-1.98350184606724110 .4829911819814661$ C $0.8694294149811221-2.94106475198854380 .2993464190874492$ C $0.2741225624049253-4.25856378214866640 .4386913239198912$ C - $1.0443357682008443-4.07342427429070590 .6902651284227688$ C - $1.2749419686073031-2.63931558250936420 .7116556211671720$ N - 1.71739843793101850 .19372643765852550 .7141322114287882 C -2.6607147576448877 -0.78607373977693850 .8939665947197775$ C -3.9677861154117600 -0.1759549128075319 1.0583562160842179 C -3.79477316471537661 .16414216152578590 .9620398844933639$ C - 2.37940372652397741 .39481382706106590 .7358033473235733 N 0.39317013712718751 .83605890210467870 .1770296761897152 C -0.5784269604611213 2.7936767703121257 0.3031614911350609 C 0.00404175919710294 .10720119146152740 .0926757785746792 C $1.32167606430872293 .9200701301643077-0.1613043372123429$ C $1.56498207599243602 .4893169641553023-0.1122551160010142$ N $2.75629428290691841 .9502616634807648-0.3326445354870252$ N $2.1509525077303007-2.75204248886083520 .0162207969864875$ N -2.4698922128705219-2.0979712717556795 0.9109804008924538 N - 1.86453185962900532 .60469120886310890 .5658837171551307 Si $0.1598121800898382-0.07274615120456090 .3794490900176569$ C $0.6871143035427831-0.00740152687434862 .2603056219499886$ H $1.3553900624624817-0.86255916083207242 .4292738719824984$ H 1.31946937600628190 .87950265911984172 .3941008010386442 C -0.3829179987667021 -0.0324225373240310 3.3631947639491822 H - 1.05125672019649260 .83126940974191813 .2840502519161516 H - $1.0177118388230324-0.91855842421093203 .2460318697075077$ C $0.1698331283172957-0.09336606817539804 .7893787945162618$ H -0.6420488135473731 -0.1911107106563000 5.5108209371783330 H $0.8467663185115193-0.94156027494522574 .9062942459929237$ S 1.10164039224754021 .45664496314235685 .2147253714429240 H 1.20381404151423891 .22360721415967616 .5405535594973463 O - $0.2522340980375519-0.1814636097859745-1.3108540544656069$ Si $0.13763688504206510 .0801432120329894-2.8803588674630047$ H $1.2988401052892449-0.7688355992453082-3.2933007233433291$ H $0.56641669692609711 .4982093620011108-3.0908256450458844$ C - $1.3282794602401293-0.3060238211387265-3.9918924258319688$ H - $1.6480348935180236-1.3366184304496651-3.7880434976536765$ H - $2.16881958064619960 .3341045025628749-3.7034923190420570$ C - $1.0458582166684653-0.1412973226309903-5.4944772356642915$ H $-0.2329264649798375-0.8108657739988400-5.7993436173721182$ $\mathrm{H}-0.69146917733945270 .8770012022865590-5.7035855910775783$ C -2.2560224389318986 - $0.4266399595758683-6.3911100296122632$ H - $1.9243867315574008-0.4325823446147478-7.4411485238747819$ H - $2.6359184007161076-1.4307818713076668-6.1738712802474671$ N -3.3476310128036908 $0.5320521967500019-6.1476416068078539$ H -3.0626476780393945 1.4450799283216575 -6.4942645980591944 H -4.1527097872682335 $0.2681286469390357-6.7089277993374452$ H $5.14074955828100320 .5806051017058798-0.7950008339827731$ H $4.7919042396500373-2.1108360733108595-0.5990675134681733$ H $0.8279079120598634-5.18068758325528030 .3396034530463422$ H - $1.8196732795304797-4.80885484047944000 .8473815055120518$ H $-4.8759007887148442-0.73839728963239181 .2191598414580975$ H - 4.52829008305085261 .95466844983901861 .0235283465165816 H - 0.55885165801421245 .02785043603003870 .1400901078513175 H 2.08741320108316544 .6517651288988713 -0.3732969163090914 